cular dystrophy, provide superb analyses of mortality and morbidity information as well as reviewing the aetiology of these conditions. The authors do not perhaps deal quite as well as they should with the problems of mortality certification in multiple sclerosis but they give one of the best descriptions I have read of the importance of epidemiological studies of migration in disentangling possible cause. The studies on epilepsy and other convulsive disorders give a most comprehensive review of the epidemiology of these conditions and demonstrate the value of mortality and morbidity statistics. There is also a reasonable analysis of diagnostic and other problems in this context.

The latter chapters are disappointing and highlight the difficulty of writing definitive works on such a wide variety of conditions. The chapters on Down's syndrome, congenital malformations and facial nerve palsy make no mention of possible aetiological factors and do not attempt to discuss the ways in which these conditions may arise. In the review of Down's syndrome, for example, nothing is said about the maternal age factor, the difference in aetiology between Down's syndrome in the offspring of young and old mothers, nor about the association between Down's syndrome and leukaemia.

With these reservations, the book is outstanding. WALTER W. Holland

\section{Sand surfaces}

Atlas of Quartz Sand Surface Textures. By David Krinsley and John C. Doornkamp. (Cambridge Earth Science Series.) Pp. $\mathrm{x}+91$. (Cambridge University: London, July 1973.) £6.50; $\$ 19.50$.

A METHOD of environmental analysis which depends upon descriptions of grain surface textures seen under the electron microscope from a wide variety of known environments has the inherent disadvantage that it requires a considerable accumulated experience and cannot yield results by a few simple measurements. Thus, the technique has largely been used by those prepared to invest a large amount of time, an investment which has seemed unlikely to be repaid by the results obtained. Krinsley and Doornkamp have now tried to make their experience available to those who might simply wish to use the technique as one of several means of analysis rather than embark upon an ambitious research programme. To do this, they have assembled a series of 122 excellent photographs which they claim to represent the principal associations of textures which, when taken together, could be used to characterise an environment.

Instead of the potential user having to undertake an exhaustive search of the literature to construct for himself a pattern of variation, this is now done for him by the authors. The book also contains an introduction to the transmission and scanning electron microscopes, and their development and use in these studies, the preparation of samples; principles of interpretation; a particularly useful diagram showing the frequency of certain types of structures from a variety of environments; and a complete bibliography of the field.

It is clear that the book cannot be used directly as a key to environment, as it is the associations of textures which are important. The statistical analysis which this implies is not necessarily as easy as it may seem, for the number of clearly differentiated surface features which could be used in such an analysis are few. This leads to the general criticism that although analysis is based on descriptions of surface features, these are often vague and over-generalised, and often difficult to follow, such as the description on page 14 of mechanical $\mathrm{V}$-shaped patterns. For example, although abundant reference is made to upturned plates, this term seems to cover a broad variety of forms which could usefully be differentiated.

For young research workers looking for rewarding topics, the book immediately suggests two. The possibility of quantified analysis; and the experimental reproduction of textures which might enable us to identify the processes at work and thus enable us better to specify the nature of the environment. The authors' own structural interpretations are sometimes unconvincing. But this lucid exposition in such a useful form will persuade many to use a technique which they may have previously regarded with awe or suspicion, and should find its way on to the bookshelves of most sedimentologists.

\section{G. S. Boulton}

\section{Fighting malaria}

Malaria: The Design, Use and Mode of Action of Chemotherapeutic Agents. By R. M. Pinder. Pp. 336. (Scientechnica: Bristol, September 1973.) $£ 10$.

THE preface of this book states that malaria remains a formidable global problem and that it is still the major killing disease of the tropics. One could not disagree with this statement even though the achievements of malaria eradication in many countries outside the truly tropical belt should not be underestimated. The partial failure of global malaria eradication is not due solely to the resistance of malaria parasites to drugs or to insecticide resistance of Anopheles vectors of the disease. This oversimplified explanation of a complex situation cannot be easily accepted by informed readers.
The book is composed of two unequal and rather unbalanced parts. Out of nine chapters six are devoted to chemotherapy of malaria; they comprise the testing and evaluation of antimalarials, a summary of the present status of various drugs, an outline of newer approaches to malaria chemotherapy, an account of miscellaneous compounds, including many experimental ones, a discussion of the mode of action of antimalarials and the review of the present status of drug resistance in malaria.

It is obvious that the author has had access to a formidable collection of reprints and index cards. And yet I missed in this part of the book an attempt at critical analysis of the laboriously collected information. It is as if anything that has been published were of equal importance just because it appeared in print. This outline of problems of chemotherapy of malaria supported by numerous references is, however, of some value although it cannot match the excellence and authoritativeness of W. Peters's Chemotherapy and Drug Resistance in Malaria published in 1970.

The remaining three chapters of the book must have been written as an afterthought, perhaps to justify the allembracing main title which overshadows the more appropriate subtitle. The chapter dealing with epidemiology of malaria gives a succinct account of the progress of malaria eradication and of its reverses; this is followed by a brief review of entomological and parasitological aspects. A chapter on malaria parasites includes the life cycle, the symptoms of clinical infection, and a notion of immunity. It is here that the author's nodding acquaintance with many aspects of malariology is evident. There are many statements that are either half true or quite wrong. Thus, neither pyrimethamine nor chloroquine has been "massively" used in common salt and the number of projects with medicated salt was small (pages 16-17). $P$. falciparum and $P$. vivax do not occur in higher apes in Africa (page 35). The population of tropical Africa is not infected in toto by quartan malaria (page 36). Sulphonamides are not causal prophylactic drugs (page 40).

The last chapter entitled "Malaria, the Way of the Dodo?" has also a quota of errors and infelicities (such as reference to the "wily plasmodium") that would be tedious to quote; however, the possibility of altering the manbiting habit of anopheline mosquito visualised by the author as a method of malaria eradication indicates his fleeting acquaintance with the discipline of malariology and with the realities of malaria control. To a question of for whom this book has been written I can give no satisfactory answer.

L. J. BRUCE-ChWATt 